\section{Stellenwert und Umfang der phlebologischen Versorgung in dermatologischen Praxen}

\author{
R. Sommer ${ }^{1}$, R. von Kiedrowski ${ }^{2}$, T. Stavermann ${ }^{3}$, R. Reinert ${ }^{1}$, N. Kirsten ${ }^{1}$, M. Augustin ${ }^{1}$ \\ ${ }^{1}$ Institut für Versorgungsforschung in der Dermatologie und bei Pflegeberufen (IVDP), Universitätsklinikum Hamburg-
} Eppendorf (UKE); ${ }^{2}$ Dermatologische Spezialpraxis, Selters; ${ }^{3}$ Dermatologische Praxis, Berlin tienten pro Woche, entsprechend 880 pro Jahr. Häufigste Leistungen: Venendiagnostik (Doppler/Duplex) und Verödungen von Besenreisern oder Seitenästen (jeweils $>50 \%$ ). $43 \%$ zeigten sich mit ihren phlebologischen Behandlungsmöglichkeiten eher oder sehr zufrieden, $35 \%$ schätzten die Versorgungssituation in der Phlebologie schlechter als vor 10 Jahren ein. Der Zusatzweiterbildung wird von $82 \%$ eine hohe Bedeutung für das Fach zugesprochen. Fazit: Die phlebologische Versorgung, wie auch die Zusatzbezeichnung „Phlebologie", ist für die Dermatologie von großer Bedeutung.

Importance and extent of phlebological health care in dermatological practices Phlebologie 2018; 47: 252-256 https://doi.org/10.12687/phleb2441-5-2018

Eingegangen: 05. Juli 2018

Angenommen: 19. Juli 2018

English version available at: www.thieme.de/phlebo

\section{Keywords}

Health care, phlebology, survey, qualification, certification

\section{Summary}

Background: Venous diseases are of great socio-economic importance in Germany. Question: What is the scope and importance of phlebological care by office-based dermatologists? Methods: Structured online survey in May 2018 in $n=1,500$ dermatological practices nationwide. Results: Of the 704 (46.9\%) practices, $49.7 \%$ have additional certified qualification in phlebology. $87 \%$ of dermatologists carry out phlebological treatments, $74 \%$ of which are based on contracts with the statutory health insurances. On average they treat 18 phlebological patients per week and 880 per year, respectively. Most frequent procedures: Vein diagnostics (Doppler/duplex) and sclerosis of spider veins or lateral branches (each $>50 \%$ ). $43 \%$ were rather or very satisfied with their phlebological treatment options, 35\% assessed the care situation in phlebology worse than 10 years ago. Certified qualification in phlebology is considered to be of great importance by $82 \%$. Conclusion: Specialized phlebological care as well as the additional certificate "phlebology", are mostly considered as important for dermatology as a specialty.

\section{Hintergrund}

Phlebologische Erkrankungen spielen in Deutschland wie auch in anderen westlichen Ländern eine große Rolle (1). Sie sind durch einheitliche Kriterien in Klassifikation und Therapie für die Versorgung gut standardisiert $(2,3)$. Schwerpunktversorger sind Ärzte mit Zusatzqualifikation in Phlebologie. Für diese wurde 1993 die Zusatzweiterbildung „Phlebologie“ eingeführt (4).

Die Versorgungsnotwendigkeit der peripheren Venenerkrankungen ist hoch. In Deutschland ist von etwa 15-20\% behandlungsbedürftiger Prävalenz in der Bevölkerung auszugehen (5), im höheren Alter von über 80 Jahren liegt sie bei über 70\% (6). Der Versorgungsbedarf resultiert dabei sowohl aus den patientenseitigen Belastungen (7-10) als auch ihrer ökonomischen Bedeutung, insbesondere bei fortgeschrittenen Stadien $(11,12)$. 


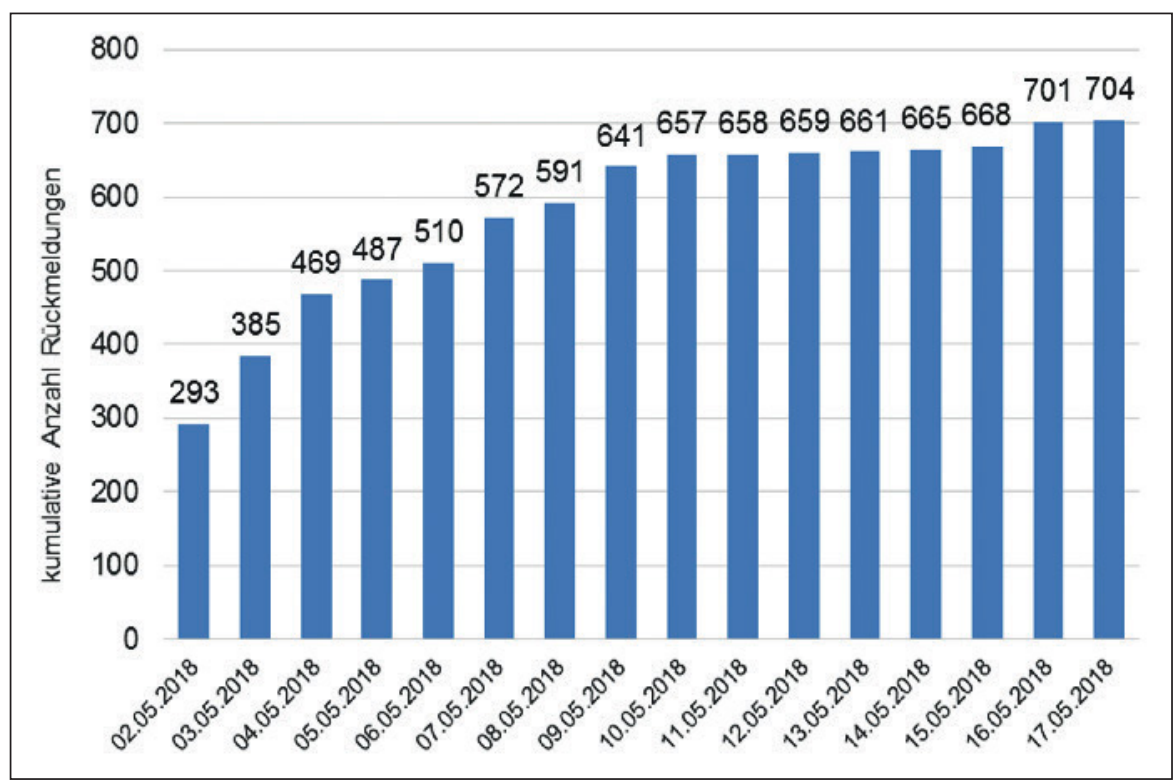

Tab. 1 Leistungsmerkmale der dermatologischen Praxen $(\mathrm{n}=704)$

\begin{tabular}{l|l|l|}
\hline \multicolumn{2}{|l}{ Anzahl phlebologischer Leistungen/Quartal } \\
\hline Mittelwert & 18,0 \\
\hline Standardabweichung & 25,4 \\
\hline Median & 10 \\
\hline Max & 250 \\
\hline Min & 0 \\
\hline Zusatzbezeichnung „Phlebologie“ \\
\hline & $n$ \\
\hline Ja & 333 \\
\hline Nein & 337 \\
\hline keine Angabe & 34 \\
\hline
\end{tabular}

Abb. 1 Inklusionsverlauf der rückmeldenden Praxen $(n=704)$

Abb. 2

Häufigkeit der phlebologischen Leistungen in den dermatologischen Praxen $(\mathrm{Pa}$ tienten pro Quartal $n=660$ )

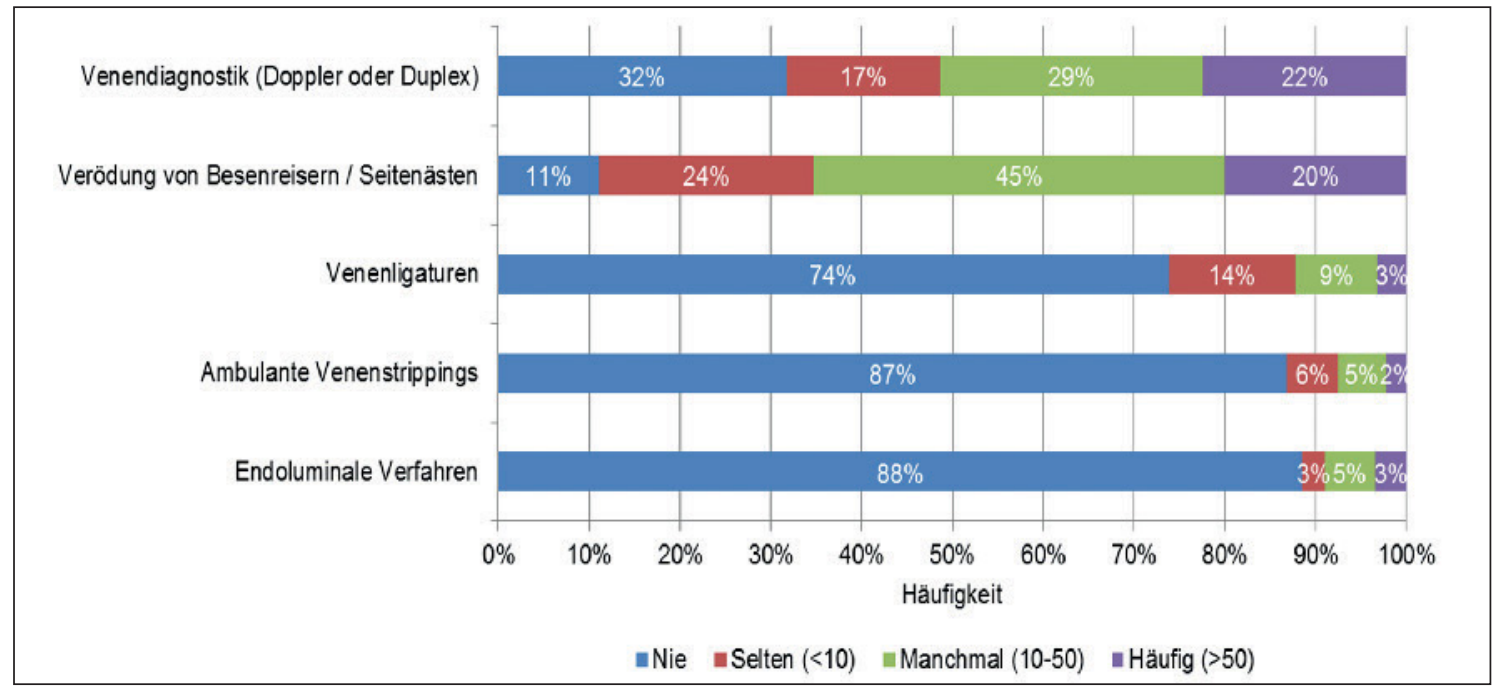

\section{Fragestellungen}

1. Wie häufig wird in den dermatologischen Praxen die Zusatzweiterbildung "Phlebologie“ geführt?

2. Welche Leistungen werden erbracht?

3. Welche Bedeutung hat die phlebologische Versorgung für die niedergelassenen Dermatologen?

\section{Methoden}

\section{Studiendesign}

Die Merkmale der dermatologischen phlebologischen Versorgung wurden mit einer strukturierten Onlineumfrage vom 03. bis
07.05.2018 unter den niedergelassenen Dermatologen (ca. 1500 Praxen) in Deutschland ermittelt. Dieser Survey erhob den Anteil an Dermatologen mit der Zusatzweiterbildung „Phlebologie“ sowie die damit verbundenen Leistungskomponenten. Begleitet wurde der Fragebogen mit einem Anschreiben der Studienleiter und des Berufsverbandes der Deutschen Dermatologen.

Die statistische Analyse erfolgte deskriptiv unter Einsatz von SPSS für Windows, Version 23.

\section{Ergebnisse}

\section{Teilnehmende Praxen}

Zur beispielhaften Abbildung der aktuellen Versorgungssituation wurde vom 03.05. bis 17.05.2018 ein Onlineumfrage in 1500 dermatologischen Praxen in Deutschland durchgeführt. Die Versendung erfolgte durch den Server des Berufsverbandes der Deutschen Dermatologen (BVDD). Auswertbare Antworten wurden innerhalb der Erhebungsphase von 704 Dermatologen eingereicht, dies entspricht einer Teilnahmequote von $46,9 \%$ ( $>$ Abb. 1 ). 


\section{Ambulante Versorgung durch Dermatologen}

87\% der Dermatologen führen in ihrer Praxis phlebologische Behandlungen durch, $74 \%$ leisten dies im Zuge der ver- tragsärztlichen Versorgung ( $\triangleright$ Tab. 1$)$. Im Durchschnitt versorgen sie 18 Patienten mit phlebologischen Beschwerden pro Woche, entsprechend etwa 880 pro Praxis und Jahr. Die häufigsten erbrachten Leistungen sind dabei die Venen-
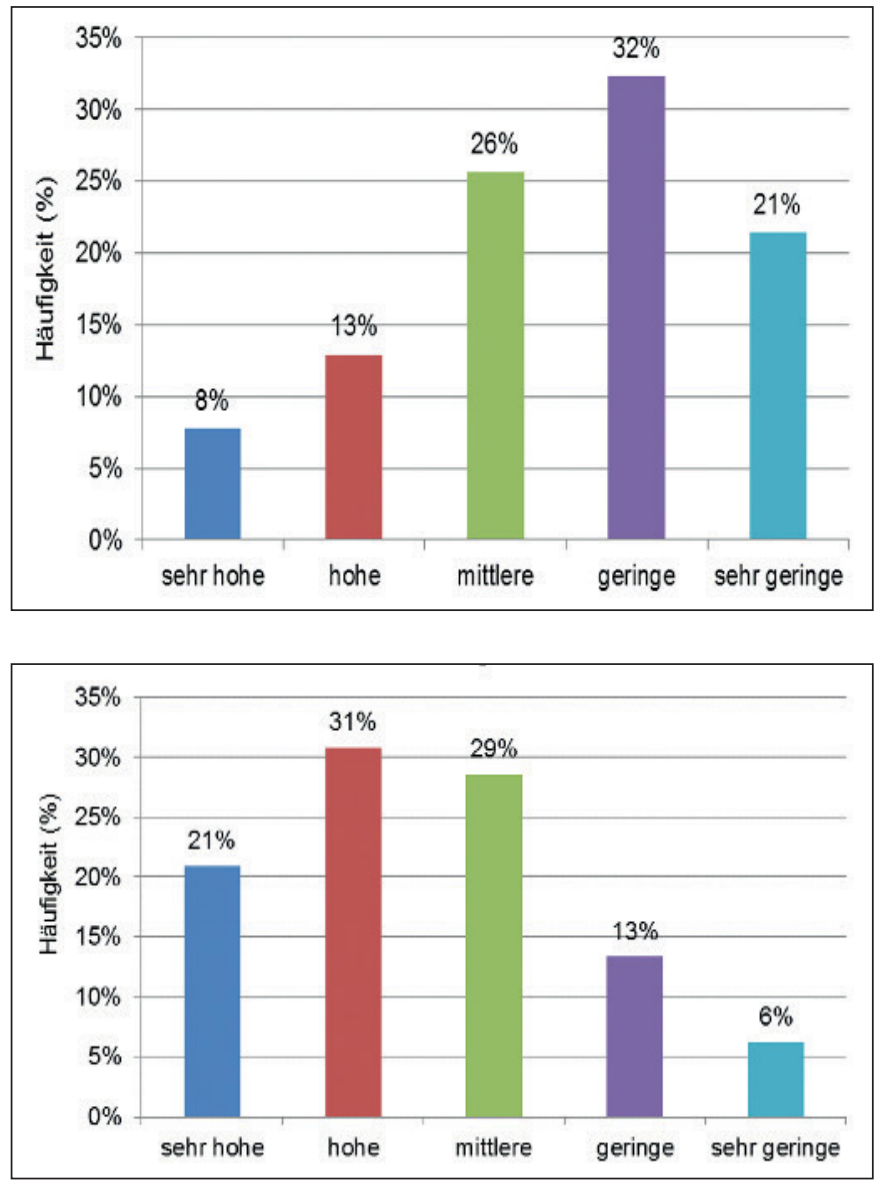

Abb. 3

Bedeutung der phlebologischen Versorgung in den dermatologischen Praxen $(n=659)$

Abb. 4

Bedeutung der Zusatzbezeichnung „Phlebologie" für die Dermatologie aus Sicht der niedergelassenen Dermatologen $(n=658)$ diagnostik (Doppler oder Duplex) und Verödungen von Besenreisern oder Seitenästen (jeweils über 50\%; A Abb. 2).

In 49,7\% der Praxen hat mindestens ein Kollege die Weiterbildung „Phlebologie“ erworben. Für 47\% der dermatologischen Praxen hat die Phlebologie eine sehr hohe, eher hohe oder mittlere Bedeutung, in 53\% eine geringe oder sehr geringe ( Abb. 3).

Der Weiterbildung „Phlebologie“ wird von $82 \%$ der Dermatologen eine relevante Bedeutung zugesprochen, davon sprechen $52 \%$ der Weiterbildung eine hohe oder sehr hohe und 29\% eine mittlere Bedeutung zu ( $\triangleright$ Abb. 4). Etwa 19\% der antwortenden Dermatologen schätzen die Bedeutung der Zusatzbezeichnung als gering oder sehr gering ein.

Die nicht oder nur teilweise versorgenden Dermatologen stehen in der Versorgung weit überwiegend mit anderen Fachgruppen im Austausch, davon stehen 34\% der Praxen mit Dermatologen, die einen Schwerpunkt in Phlebologie haben, $41 \%$ mit Chirurgen und 43\% mit Angiologen im Austausch ( Abb. 5b). Bezüglich der stationären Versorgung geben ca. 51\% der dermatologischen Praxen eine Kooperation mit einer dermatologischen Abteilung, $46 \%$ mit einer chirurgischen, $27 \%$ mit einer angiologischen und $17 \%$ mit einer spezialisierten phlebologischen Fachklinik an $(\triangleright$ Abb. 5b).

$43 \%$ zeigten sich mit ihren phlebologischen Behandlungsmöglichkeiten eher oder sehr zufrieden, $34 \%$ teils, teils, $22 \%$

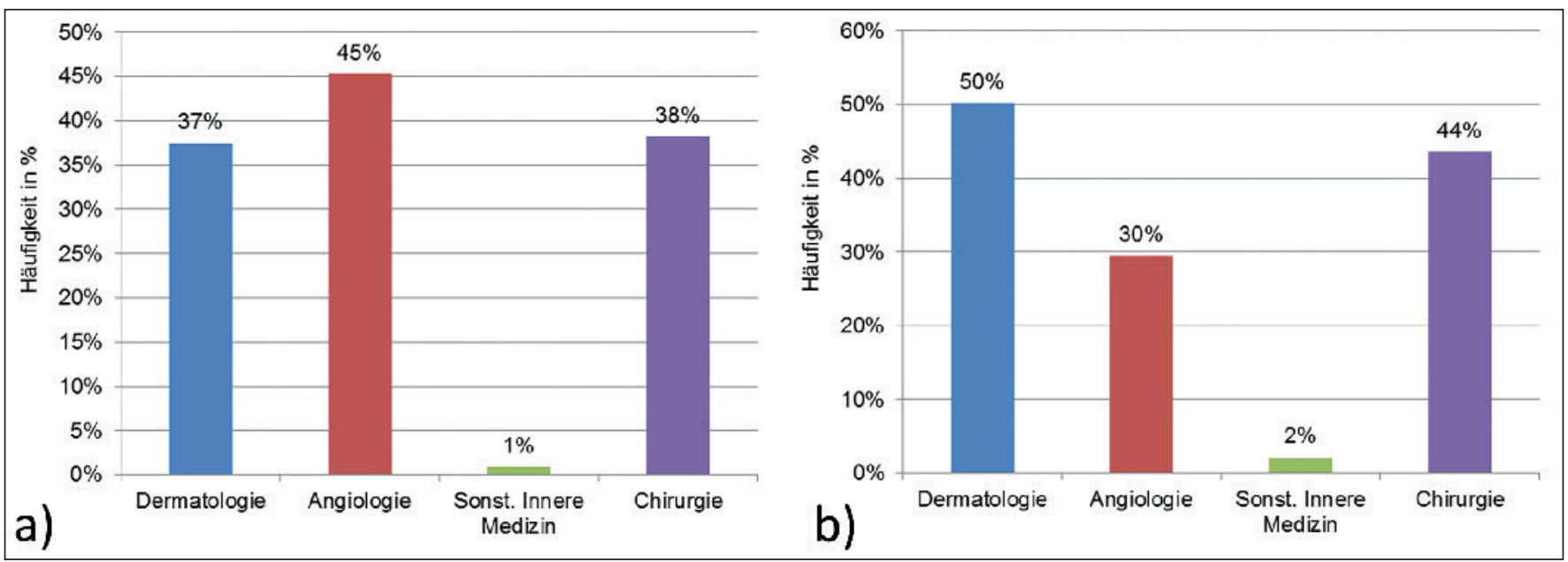

Abb. 5 Anteil der üblichen Überweisungen in ambulante (a) oder stationäre (b) phlebologische Weiterversorgung durch niedergelassene Hautarztpraxen $(n=654)$ 


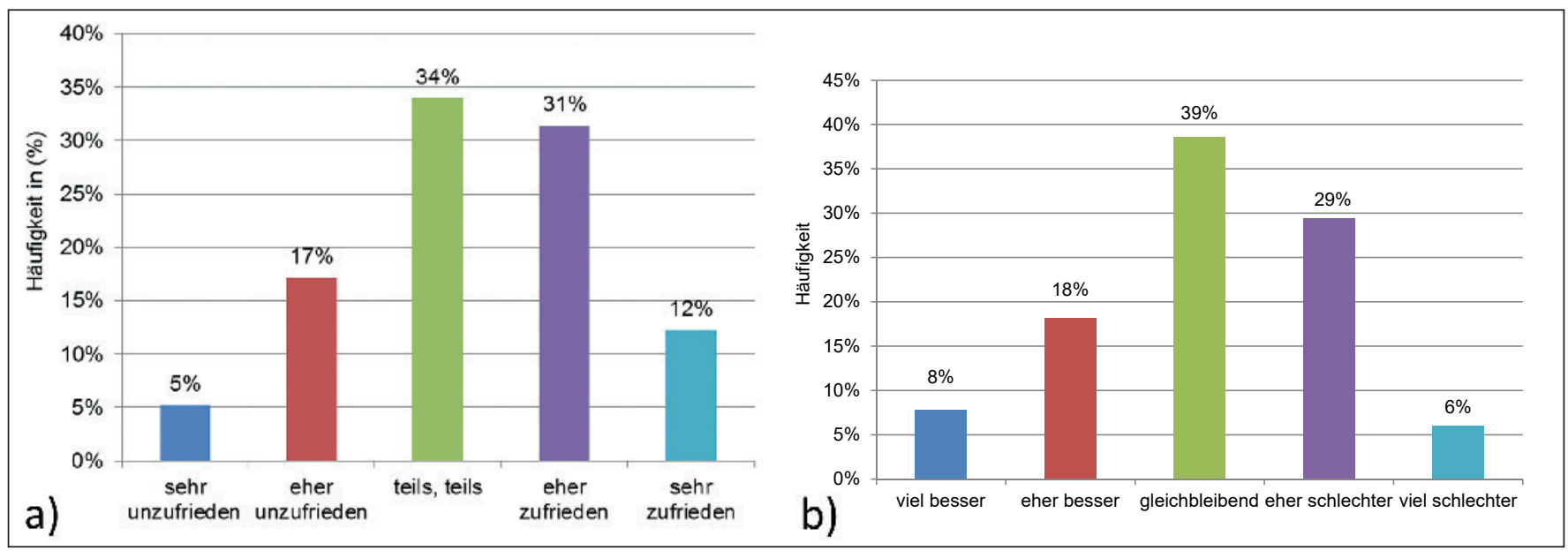

Abb. 6 Zufriedenheit der niedergelassenen Dermatologen mit ihren phlebologischen Behandlungsmöglichkeiten (a) und Einschätzung der aktuellen Versorgungssituation im Vergleich zur Situation vor 10 Jahren (b) (je $n=653$ )

eher oder sehr unzufrieden ( $\triangleright$ Abb. 6). Häufigste Gründe für Unzufriedenheit waren die als unzureichend bewerteten Vergütungen für die qualitätsgesicherte phlebologische Versorgung.

In der Zusammenschau spielt die phlebologische Versorgung wie auch die $\mathrm{Zu}$ satzbezeichnung „Phlebologie“ für etwa die Hälfte der deutschen dermatologischen Praxen eine Rolle und wird auch in der Versorgung wahrgenommen ( Tab. 2). Dabei kommen in erster Linie kleine phlebologische Eingriffe zur Anwendung. Die größeren ambulanten Eingriffe beschränken sich auf weniger und stärker spezialisierte dermatologische Praxen.

\section{Diskussion}

Ziel der vorliegenden versorgungswissenschaftlichen Studie war die Erfassung des Aufkommens von phlebologischer Versorgung durch dermatologische Praxen in Deutschland. Erhoben werden sollten ferner die Leistungsmerkmale und die Einschätzungen der Dermatologen zur phlebologischen Versorgung. Mit den vorliegenden Daten zeichnet sich ab, dass die spezialisierte phlebologische Versorgung durch einen relevanten Teil der dermatologischen Praxen, jedoch bei weitem nicht von allen, durchgeführt wird. Ein relevanter Teil führt zudem eher kleinere Eingriffe durch, sodass die Vollversorgung mit einem breiten Feld von Interventionen einer kleineren Zahl spezialisierter Praxen vorbehalten ist. Dementsprechend weisen die meisten der nicht spezialisierten Praxen ein regelmäßiges Zuweisersystem zu phlebologischen Praxen und Klinikabteilungen auf. Dieser Spezialisierungsgrad wird zukünftig weiter steigen, da die meisten dermatologischen Praxen inzwischen andere, unterschiedliche Versorgungsschwerpunkte haben, etwa die Tumorbehandlung oder die Versorgung der chronisch-entzündlichen

Dermatosen. In dieser Situation zunehmender Schwerpunktbildung muss die Erkennbarkeit und Qualitätssicherung der Praxen mit phlebologischem Schwerpunkt gewährleistet sein. Dementsprechend hat die Bundesärztekammer im Jahr 2018 die Zusatzbezeichnung „Phlebologie“ in ihrem Katalog belassen. Die Zahl der in Deutschland tätigen Ärzte mit der Zusatzbezeichnung „Phlebologie“ liegt bei etwa 3400 (4). Diesen Spezialisten kommt die Aufgabe zu,

Tab. 2

Synopsis: Eckdaten der versorgenden dermatologischen Praxen in der phlebologischen Versorgung

\begin{tabular}{|l|l|}
\hline Versorgung & $\%$ \\
\hline Praxen mit phlebologische Versorgung, gesamt & 87 \\
\hline Praxen mit vertragsärztlicher phlebologischer Versorgung & 74 \\
\hline Praxen mit Zusatzweiterbildung „Phlebologie” & 55 \\
\hline Praxen mit Venendiagnostik & 52 \\
\hline Praxen mit Verödungen von Besenreisern / Seitenästen & 67 \\
\hline Praxen mit Venenligaturen oder -strippings & 8 \\
\hline Praxen mit endoluminalen Verfahren & 9 \\
\hline Patienten pro Jahr & $\mathrm{n}=883$ \\
\hline Einstellungen & $\%$ \\
\hline Phlebologie hat eher hohe/sehr hohe Bedeutung für die Praxis & 49 \\
\hline Zusatzweiterbildung Phlebologie wichtig für die Versorgung & 82 \\
\hline Kooperation mit... & $\%$ \\
\hline Dermatologen mit Schwerpunkt Phlebologie & 34 \\
\hline Chirurgen & 41 \\
\hline Angiologen & 43 \\
\hline Dermatologischen Fachabteilung & 51 \\
\hline Chirurgische Fachabteilung & 46 \\
\hline Angiologische Fachabteilung & 27 \\
\hline
\end{tabular}


zu einer höheren Qualität der phlebologischen Versorgung selbst, oder durch Wissensvermittlung an die basisversorgenden Ärzte, beizutragen. Die Dringlichkeit der qualitätsverbessernden Maßnahmen in der Versorgung wird besonders deutlich, wenn die Versorgungsdaten in Betracht gezogen werden. Hier gibt es insbesondere bei höhergradiger Veneninsuffizienz noch erhebliche Defizite, etwa beim Einsatz der Kompressionstherapie im ländlichen Raum (8, 13). Selbst in Fachkreisen gibt es Nachholbedarf in der praktischen Kompetenz der Venenbehandlung mittels Kompressionssystemen (14). Auch für die Qualitätssicherung in der breiteren Versorgung erscheint das Setzen von Anreizen für mehr phlebologische Expertise bei den Dermatologen wie auch bei anderen Fachrichtungen geboten. Der große Nutzen einer phlebologischen Expertise liegt in der Verbesserung der dermatologischen Versorgung, da auch eine Vielzahl dermatologischer Konditionen, wie Stauungsekzeme oder durch Veneninsuffizienz aggravierte Dermatitiden, erfasst werden.

\section{Interessenkonflikt}

Nach Angaben der Autoren bestehen keine Interessenkonflikte.

\section{Ethische Richtlinien}

Für das Manuskript wurden keine Studien an Menschen oder Tieren durchgeführt.

\section{Literatur}

1. Augustin M, Sommer R, Zander N et al. Epidemiologie, Versorgungsbedarf und Versorgungsvolumina peripherer Venenkrankheiten in Deutschland: Multi-Source Analyse von Primär- und Sekundärdaten. Phlebologie 2018. doi: 10.12687/phleb2440-5-2018.

2. Eklöf B, Rutherford RB, Bergan JJ et al. Wakefield, Revision of the CEAP classification for chronic venous disorders: Consensus statement. Journal of Vascular Surgery 2004;40:1248-1252.

3. Stücker M, Debus ES, Hoffmann J et al . Consensus statement on the symptom-based treatment of chronic venous diseases. J Dtsch Dermatol Ges 2016; 14:575-83.

4. GBE-Bund. http://www.gbe-bund.de/oowa921-in stall/servlet/oowa/aw92/dboowasys921.xwdevkit/ xwd_init?gbe.isgbetol/xs_start_neu/ \&p_aid=i\&p_aid $=33954832 \&$ nummer $=612 \& p \_s$ prache=D\&p_indsp=93\&p_aid=42395654. Zugriff 30.04.2018

5. Rabe E, Pannier-Fischer F, Bromen K et al. Bonner Venenstudie der Deutschen Gesellschaft für Phlebologie - Epidemiologische Untersuchung zur Frage der Häufigkeit und Ausprägung von chronischen Venenkrankheiten in der städtischen und ländlichen Wohnbevölkerung. Phlebologie 2003; 32: 1-14.

6. Kemper C, Sauer K, Glaeske G. Barmer GEK Heilund Hilfsmittelreport 2011. Auswertungsergebnisse der BARMER GEK Heil- und Hilfsmittel- daten aus den Jahren 2009 bis 2010. Schriftenreihe zur Gesundheitsanalyse, Band 10, 2011.

7. Augustin M, Debus ES, Bruning G et al. Development and Validation of a Short Version of the Freiburg Life Quality Assessment for Chronic Venous Disease (FLQA-VS-10). Wound Medicine 2015; 8: 31-35.

8. Zschocke I, Bross F, Maier K et al. Quality of Life in Different Stages of Chronic Venous Insufficiency and Leg Ulcer. Dermatol Psychosom 2002; 3: 126-131.

9. Augustin M, Dieterle W, Zschocke I et al. Development and Validation of a Disease Specific Questionnaire on the Quality of Life of Patients with Chronic Venous Insufficiency. Vasa 1997; 26: 291-301.

10. Sommer R, Augustin M, Blome C. Erfassung von Lebensqualität und Verwendung patientenberichteter Endpunkte in der Praxis. Phlebologie 2017; 46: 334-339.

11. Purwins S, Herberger K, Debus ES et al. Cost-ofillness of chronic leg ulcers in Germany. Int Wound J 2010; 7: 7-102.

12. Augustin M, Brocatti LK, Rustenbach SJ et al. Cost-of-illness of leg ulcers in the community. 2014; 11: 283-92.

13. Heyer K, Protz K, Glaeske G, Augustin M. Epidemiology and Use of Compression Treatment in Venous Leg Ulcers: Nationwide Claims Data Analysis in Germany. Int Wound J 2016; 14: 338-343.

14. Heyer K, Protz K, Augustin M. Compression Therapy - Cross-sectional Observational Survey About Knowledge and Practical Treatment of Specialised and Non-specialised Nurses and Therapists. Int Wound J 2017; 14: 1148-1153. 